\title{
Martin Luthers Heldenbild in Deutschland und sein transkultureller Einfluss in China ${ }^{1}$
}

\section{Lin Chunjie}

\section{Martin Luther im Diskurs}

Als Urheber der Reformation und bedeutender Autor übte Martin Luther großen Einfluss auf die Welt aus, zu Lebzeiten bis heute. Am 31. Oktober 1517 veröffentlichte er seine 95 Thesen, in denen er vor allem die Ablässe der römischkatholischen Kirche ablehnte. Dies führte zur Reformation, der Spaltung der christlichen Kirche und der Entstehung der evangelischen Kirchen. Martin Luther übersetzte die Bibel ins Deutsche und sein Beitrag zur Entwicklung der deutschen Sprache ist unerreicht. Dennoch ist er bis heute eine kontrovers diskutierte historische Persönlichkeit.

\subsection{Forschungsstand}

Seit der Reformation ist das Bild Martin Luthers in der öffentlichen Wahrnehmung ständigem Wandel unterzogen, seinem Werk und Wirken gilt ungebrochenes Forschungsinteresse. Walther von Loewenich und Hubert Jedin studierten die Wandlungen des Lutherbildes in der evangelischen ${ }^{2}$ bzw. katholischen ${ }^{3}$ Kirchengeschichte. In der DDR veröffentlichte Gordon R. Mork 1983 seine Abhandlung Martin Luther's Left Turn: The Changing Picture of Luther in East German Historiography. ${ }^{4}$ Schwerpunktmäßig wird in der Forschung Martin Luthers Status als Held untersucht. So etwa von Hartmut Lehmann 1984, ${ }^{5}$ von Robert Kolb $1999^{6}$ und von Henrike Holsing, die ihre Dissertation diesem Thema widmete. ${ }^{7}$ In China

1 Gefördert vom National Social Science Fund of China: „Study of the Chronicle of Martin Luther" (Project No. 16CSS001).

2 Walther von Loewenich: Wandlungen des Evangelischen Lutherbildes im 19. und 20. Jahrhundert, in: Karl Forster (Hg.): Wandlungen des Lutherbildes, Würzburg 1956, S. 49-76.

3 Hubert Jedin: Wandlungen des Lutherbildes in der Katholischen Kirchengeschichtsschreibung, in: Karl Forster (Hg.): Wandlungen des Lutherbildes, Würzburg 1956, S.77-101.

4 Gordon R. Mork: Martin Luther's Left Turn: The Changing Picture of Luther in East German Historiography, in: The History Teacher 16, 1983, S. 585-595.

5 Hartmut Lehmann: Martin Luther als Deutscher Nationalheld im 19. Jahrhundert, in: Luther 55, 1984, S. 53-65.

6 Robert Kolb: Martin Luther as Prophet, Teacher, Hero. Images of the Reformer, 15201620, Grand Rapids 1999.

7 Henrike Holsing: Luther - Gottesmann und Nationalheld. Sein Image in der deutschen Historienmalerei des 19. Jahrhunderts, Köln 2004. 
hat in jüngerer Zeit Zhang Ke die Veränderung des Lutherbildes im China der späten Qing-Dynastie untersucht. ${ }^{8}$

\subsection{Widersprüchliche Bilder von Luther}

In der katholischen Kirche betrachtete man Luther als „den Zerstörer der Kircheneinheit, den skrupellosen Demagogen und frechen Revolutionär, der durch seine Häresien unzählige Seelen ins Verderben gestürzt, unendliches Elend über Deutschland und die ganze Christenheit gebracht" habe. ${ }^{9}$ Das Luthertum und protestantische Deutschland bewerteten sein Wirken diametral entgegengesetzt und sahen in Luther „,den Erneuerer der reinen Lehre“. ${ }^{10}$ Der Pietismus bewertete Luther als Vertreter der Herzensfrömmigkeit, als Propheten und Heiligen. „Die Aufklärung sieht in Luther vor allem den Vorkämpfer für das Recht der Vernunft und für die Gewissensfreiheit." ${ }^{11}$ Johann Gottfried Herder beurteilte Luther in seinen Briefen zu Beförderung der Humanität so: „Luther war ein patriotischer großer Mann. Als Lehrer der deutschen Nation, ja als Mitreformer des ganzen jetzt aufgeklärten Europa ist er längst anerkannt; auch Völker, die seine Religionssätze nicht annehmen, genießen seiner Reformation Früchte. "12 Nicht nur Herder befasste sich mit dem Reformer: „Luther als Apostel der Freiheit erscheint dann auch in der deutschen Klassik und im deutschen Idealismus. "13 In einem Gespräch mit Johann Peter Eckermann am 11. März 1832 erläuterte Johann Wolfgang von Goethe seine Sicht auf das Verdienst Luthers hinsichtlich der Weiterentwicklung des Glaubens in Deutschland. ${ }^{14}$

Gleichzeitig sollte Luthers Antijudaismus nicht vergessen werden. Während des Zweiten Weltkriegs suchte William Montgomery McGovern, Martin Luthers Einfluss auf die faschistische Ideologie nachzuweisen. Seine Studie From Luther to Hitler (1941) sieht in Luther und dem Luthertum frühe Vorläufer des Faschismus. McGovern konstatiert: Luther ,started with a plea for individual liberty and for freedom of conscience; yet his doctrines led directly to a belief in the divine right of kings and to the belief that monarchs have a right to dictate religious dogmas to the private individual." 15

Das Lutherbild erweist sich als kompliziert, vielfältig und widersprüchlich. Dieser Artikel konzentriert sich darauf, wie das Bild von Luther als Nationalheld

8 Zhang Ke: Zur Veränderung des Bildes von Martin Luther im China der späten QingDynastie, in: Zeitschrift in Zhejiang 6, 2013, S. 123-129.

9 Jedin: Wandlungen (Anm. 3), S. 80.

10 von Loewenich: Wandlungen (Anm. 2), S. 51.

11 Ebd., S. 52.

12 Johann Gottfried Herder: Briefe zu Beförderung der Humanität, II, 18, J. G. H. Sämmtliche Werke, hg. von Bernhard Suphan, Bd. 17, Berlin 1881, S. 87-91, hier S. 87. von Loewenich: Wandlungen (Anm. 2), S. 54.

14 Vgl. ebd., S. 54.

15 William Montgomery McGovern: From Luther to Hitler, Cambridge 1941, S. 31. 
entstand, wie sich das Lutherbild in China ausbreitete und im chinesischen Kontext zu einem Heldenbild wurde. Der Artikel wird außerdem die Außenwahrnehmung des Leiters der Wuxu-Reform, Kang Youwei, als „Martin Luther des Konfuzianismus" $" 16$ analysieren und den transkulturellen Einfluss des Lutherbildes in China untersuchen.

\section{Das Lutherbild in Deutschland}

\subsection{Luther als Held}

Wer ist ein Held? Welchen Heldentypus brauchen wir? Helden sind die wichtigsten Vorbilder für das Volk. Ihre Entstehung reicht historisch weit zurück und ihre Konstruktion vollzog sich häufig im Wechselspiel zwischen dem Volk und den Intellektuellen. Hartmut Lehmann meinte: „Helden mußten gewissermaßen aus dem Dunkel auf die Bühne der Geschichte treten und schnell zu Rettern ihres Volkes aufsteigen. “" „Ein wahrer nationaler Held konnte nur sein, wer eine Ära des Niedergangs, der Korruption und der Scham im Leben seines Volkes beendete und eine neue Ära des Glanzes, der Ehre und des Stolzes einleitete." 18

Luther wurde im 16. und 17. Jahrhundert von den Protestanten als prophetischer Held betrachtet. „Luther the prophetic hero served the purpose of rallying his latter-day followers to the cause of Protestant or evangelical independence from Roman control." ${ }^{\prime 19}$

Im ausgehenden 18. und im frühen 19. Jahrhundert fanden heftige Auseinandersetzungen zwischen der romanischen und germanischen Welt statt. Frankreich war der Hauptfeind Deutschlands und ein Vertreter des Katholizismus. Preußen und Österreich wurden im Napoleonkrieg von Frankreich besiegt und das Heilige Römische Reich Deutscher Nation fiel im Jahr 1806. Diese Situation trug ihren Teil zum Erstarken des deutschen Nationalismus bei. Martin Luther wurde im 19. Jahrhundert als Nationalheld präsentiert, als Vorkämpfer für Deutschlands Einheit und gegen alles Fremde, der durch seine Bibelübersetzung in die deutsche Sprache einem deutschen Nationalbewusstsein buchstäblich neue Worte gegeben hatte. Er wurde neben Persönlichkeiten wie Friedrich Barbarossa eingereiht und „als zweiter Hermann der Cherusker bezeichnet, der den deutschen Boden von römischem Einfluß befreit habe." 20

Trotz des anhaltenden Streits zwischen der evangelischen und der katholischen Kirche waren Luthers Verdienste um die neuere deutsche Sprache und Literatur

16 Liang Qichao: Biographie Kang Youweis, in: Gesamtwerk von Liang Qichao, Bd. 1, hg. von Zhang Pinxing, Peking 1999, S. 486.

17 Lehmann: Martin Luther (Anm. 5), S. 54.

18 Ebd.

19 Kolb: Martin Luther (Anm. 6), S. 101.

20 Lehmann: Martin Luther (Anm. 5), S. 61. 
jenseits aller Parteiungen unbestritten. Das 19. Jahrhundert war eine wichtige Phase für eine Heroisierung Luthers. Viele Städte errichteten Lutherdenkmäler, wie Wittenberg 1821, Möhra 1861, Worms 1868, Coburg 1883 und Dresden 1885. „Eine bildungsbürgerliche Lesegesellschaft war hier der Initiator."21 Martin Luther war ein „Held des Bürgertums“.22 Im Jahr 1917, zum 400. Jubiläum der Reformation, beurteilte der Historiker Erich Marcks Luther als „Held der Nation“ und bezeichnete die Reformation als „die größte Revolution unserer Geschichte: eine Umwälzung, wirklich, wenn es je eine gegeben hat; die seelische, die religiöse, die unendlich tief hineingriff auch in die sichtbare Welt. ${ }^{\text {"23 }}$

\subsection{Luthers Heldentaten}

Was sind Luthers Heldenzeiten oder Heldentaten? Was ist Wirklichkeit, was ist erfunden oder übertrieben? Als Heldentaten Luthers gelten üblicherweise: Der Thesenanschlag von 1517, die Verbrennung der Bannandrohungsbulle von Papst Leo X. im Jahr 1520, sein Erscheinen vor dem Kaiser in Worms 1521, die Tarnung als Junker auf der Wartburg und seine Bibelübersetzung von 1522.

Die Veröffentlichung der 95 Thesen ist von immenser Bedeutung für seinen Ruf, sie markiert den Beginn der Reformation. „In vielen Lutherdarstellungen des 19. Jahrhunderts diente Luthers Zeit im Kloster als eben jene Dunkelheit, aus der heraus Luther im Jahre 1517 wie die Sonne eines neuen Tages aufstieg und den Deutschen zum Heilsbringer wurde. “24 Wiewohl sich die Überlieferung hält, dass Martin Luther die 95 Thesen an die Tür der Schlosskirche zu Wittenberg geschlagen und damit Revolutionäres vollbracht habe, ist die Historizität des Thesenanschlags dennoch umstritten. Sicher ist, dass Luther die 95 Thesen in einem persönlichen Brief an Erzbischof Albrecht von Mainz verschickt und darin die Ablässe der römisch-katholischen Kirche scharf kritisiert hat. Darauf gab ihm Erzbischof Albrecht keine Antwort. Als Luther die Thesen schließlich veröffentliche, initiierte dies die Reformation.

Am 15. Juni 1520 unterzeichnete Papst Leo X. die Bannandrohungsbulle Exsurge Domini. Diese wurde von Luther am 10. Dezember 1520 zusammen mit kirchlichen Rechtssammlungen vor dem Wittenberger Elstertor verbrannt. Das ist historisch wohl erwiesen und verbürgt.

Am 18. April 1521 fand der Reichstag in Worms statt, in dessen Rahmen Martin Luther verhört wurde. Luthers Antwort wurde auf Lateinisch und Deutsch formuliert und ist bis heute berühmt. Luther weigerte sich, seine Werke zu widerrufen und antwortete, „Es sei denn, daß ich mit Zeugnissen der heiligen Schrift, oder mit öffentlichen, klaren und hellen Gründen und Ursachen überwunden und überwie-

Holsing: Luther (Anm. 7), S. 172.

Ebd., S. 170.

3 Herfried Münkler: Die Deutschen und ihre Mythen, Hamburg 2010, S. 194.

24 Lehmann: Martin Luther (Anm. 5), S. 55. 
sen werde [...] und mein Gewissen in Gottes Wort gefangen sei, so kann und will ich nichts widerrufen, weil weder sicher noch geraten ist, etwas wider das Gewissen zu tun. Hier stehe ich, ich kann nicht anders." 25 Der große Mut, dem Kaiser zu widersprechen, macht den Reichstag zu Worms zu einem zentralen Ereignis im Verlauf der Reformation. Hier wurde Luther zum echten Helden. Der berühmte Satz „Hier stehe ich, ich kann nicht anders“ verstärkte das Bild Luthers als Reformator und Held. Allerdings ist auch die Historizität dieser Aussage umstritten. Viele Historiker tendieren dazu, Luther die Äußerung dieses Satzes abzusprechen. Heinrich Boehmer wies nach, dass das ,sogenannte Wormser Diktum [...] in der Form: ,Ich kann nicht anders, hier stehe ich, Gott helfe mir, Amen!' allerdings schon in zwei Wittenberger Drucken aus dem Jahre 1521 auf[taucht], aber Luther, der damals auf der Wartburg saß, hat mit diesen Veröffentlichungen nichts zu tun gehabt. Die heute übliche Form findet sich zuerst in dem 1546 nach seinem Tode erschienenen zweiten Bande der Wittenberger Gesamtausgabe seiner Werke. Maßgebend für die Darstellung ist der von ihm selbst inspirierte, wohl von [Justus] Jonas herrührende lateinische Bericht Reichstagsakten II, 2, 540ff." 26 Auch Herfried Münkler zufolge habe Luther diese Sentenz in Worms wohl nicht verwendet, sie sei „später hinzufügt worden. Aber sie entspricht dem Geist und Gestus von Luthers Auftritt. Sie gibt ihm einen kraftvollen Schlusspunkt; sie ist der erste Schritt der Arbeit am Mythos. ${ }^{\text {} 27}$

Während seines Aufenthaltes auf der Wartburg übersetzte Luther in sehr kurzer Zeit das Neue Testament ins Deutsche. Diese Übersetzung spielte eine große Rolle bei der Entwicklung des Neuhochdeutschen als Schrift- und Literatursprache und ist eine der wichtigsten Errungenschaften Luthers.

Aber hat Luther auch nicht-heroisches Verhalten gezeigt? Ja, etwa mit seinen bitterbösen Bauernkriegsschriften und antisemitischen Schriften. Während des Bauernkriegs schrieb Martin Luther Wider die räuberischen und mörderischen Rotten der Bauern, worin er den Aufstand der Bauern kritisierte und die Fürsten dazu aufrief, die Bauern zu unterdrücken. 1543 verfasste er Von den Juden und ibren Lügen und 1546 Eine Vermahnung wider die Juden, worin er die Juden und das Judentum stark kritisierte, judenfeindliche Stereotype erneuerte und die Leser zu Vertreibung und Versklavung der Juden aufrief. Dies hatte erhebliche Auswirkung auf die Judenverfolgung durch die Nazis im 20. Jahrhundert.

Gemäß der Forschung von Hartmut Lehmann konzentrierten sich die Lutherbiographen des 19. Jahrhunderts auf die Phase von 1517 bis 1522 und ignorierten Luthers Leben nach 1525 weitgehend, weil sie einen idealen Nationalhelden brauchten.

25 Andrea van Dülmen: Luther-Chronik. Daten zu Leben und Werk, München 1983, S. 76.

26 Heinrich Boehmer: Der junge Luther, Leipzig 1954, S. 338.

27 Münkler: Die Deutschen (Anm. 23), S. 185. 


\section{Das Lutherbild in China}

\subsection{Frühes Lutherbild in China}

Auch in China hat sich das Lutherbild ständig gewandelt. In der zweiten Hälfte des 16. Jahrhunderts kamen die Jesuiten-Missionare nach China, wobei diese hauptsächlich die Lehren und die Geschichte der katholischen Kirche propagierten und die Streitigkeiten der Reformation und Martin Luthers aus strategischer Überlegung grundsätzlich nicht erwähnten. ${ }^{28}$

Nach Chinas Niederlage im Opiumkrieg (1840-1842) stieg das Interesse chinesischer Intellektueller an Geschichte und Geografie anderer, vor allem westlicher Länder. Als das Wissen um die Reformation China erreichte, wurde Luther zunächst als Gründer der protestantischen Kirchen rezipiert. Weil die Reformation zur Spaltung der Kirche und zu den Religionskriegen geführt hatte, musste Luther als der Gründer der protestantischen Kirchen dafür verantwortlich sein, so die Argumentationskette.

$\mathrm{Xu}$ Jiyu stellte Martin Luther in seinem Werk Einfübrung in die Weltgeografie (Yinghuan zhilïe) vor und urteilte: „Ich glaube, dass Religion die Welt retten soll. Und die Länder töten sich wegen der Spaltung der Kirche. Was halten Sie von den Gründern der Religion?" 29 In China, welches seit der Antike immer Einheit angestrebt hatte, konnte das Urteil über Luther zunächst kein gutes sein, nachdem er die Teilung der westlichen Kirchen und die Religionskriege verursacht hatte.

Nach der Einführung des Protestantismus in China im 19. Jahrhundert begann die evangelische Kirche zu missionieren und mit der katholischen Kirche zu konkurrieren. Unter den Chinesen wurden Katholizismus und Protestantismus zunehmend unterschieden. Evangelische Missionare begannen, die positiven Aspekte von Luthers Reformation und die negativen Aspekte der katholischen Kirche bekanntzumachen. Der Protestantismus oder die evangelische Kirche wurden in China ,Xin Jiao' genannt. Xin bedeutet ,neu', Jiao ,Konfession und Kirche'. Xin Jiao lässt sich also mit ,neue Kirche‘ oder ,neue Konfession` übersetzen. In diesem Gegensatzpaar symbolisiert ,Neu' den Fortschritt und ist positiv konnotiert, ,Alt ${ }^{6}$ steht hingegen für Rückständigkeit und ist eher negativ besetzt. So trug die Bezeichnung ,Xin Jiao` zu einer allmählichen Aufwertung des Protestantismus bei.

28 Zhang Ke: Katholizismus und Protestantismus in der späten Qing-Dynastie aus der Perspektive der Begriffsgeschichte, in: Studien zur Geschichte 4, 2011, S. 74.

29 Xu Jiyu: Einführung zur Geschichte und Geographie von Ländern auf der ganzen Welt (Yinghuan Zhilüe), Shanghai 2001, S. 191. 


\subsection{Das Bild Luthers als Held in China}

Damit einhergehend wurde auch die Person Luthers stetig aufgewertet und entwickelte sich vor dem Hintergrund der Reformbewegung von 1898 allmählich zu einer heroischen Figur.

Nachdem China im chinesisch-japanischen Jiawu-Krieg (1894-1895) von Japan besiegt worden war, versuchten der chinesische Kaiser Guangxu und einige Intellektuelle im Jahr 1898 China zu reformieren, um dem Niedergang Einhalt zu gebieten und das Land zu neuer Prosperität zu führen. Kang Youwei (18581927) und Liang Qichao (1873-1929) waren die führenden Köpfe hinter der Reform. Sie wollten das alte China vom Absolutismus in eine konstitutionelle Monarchie überführen. Sie brauchten Vorbilder für diese Reform und fanden diese in Zar Peter I. von Russland, im japanischen Kaiser Meiji und in Martin Luther. Die Reform, die Änderungen in Erziehungswesen, Wirtschaft und Verwaltung vorsah, scheiterte nach kurzer Zeit, weil die Kaiserinwitwe Cixi putschte, und wurde daher als ,Hundert-Tage-Reform` bekannt.

Das Lutherbild wandelte sich stark während der Wuxu-Reform 1898. Im Allgemeinen sympathisierten die Missionare mit der Wuxu-Reformbewegung von 1898. Die protestantischen Missionare bewarben Martin Luther und die Reformation aktiv als Vorbild für Chinas Reformierung, auch nach dem Scheitern der WuxuReform. Der protestantische Missionar Lin Lezhi (Young J. Allen) aus den USA übersetzte und veröffentlichte 1899 Martin Luther und die Reformation (Lude Gaijiao Jilve). „Das Ziel dieses Buches ist es, Luther, den Führer der europäischen Reformation, zu einem Vorbild für Reformen im Osten zu machen. “30 Lin Lezhi glaubte, „Luthers Reformation hatte ihren Ursprung in Deutschland und breitete sich dann auf andere Länder aus. Länder wie Deutschland, das Vereinigte Königreich und die Vereinigten Staaten, in denen der Protestantismus am weitesten verbreitet ist, werden immer stärker und die Menschen können die Freiheit genießen. "31 Italien, Spanien, Frankreich und Österreich-Ungarn waren die negativen Beispiele. Daher, so die Folgerung, sei der Protestantismus dem Katholizismus überlegen. Das Lutherbild in China begann sich zu verändern. Luther wurde zum Reformhelden und zum Vorbild für Chinas Reform, wie das Zitat von Liang Qichao in seinem Werk Zur Erneuerung des Volks (Xin Min Shuo) bezeugt: „Luther schuf erstlich die Glaubensfreiheit und förderte den Fortschritt und das Glück der Menschheit." 32 Deshalb wurde Luthers Mut, gegen den Papst zu sprechen und zu handeln, hoch bewertet und die Meinung, dass Luther ein deutscher Nationalheld sei, setzte sich auch in China durch.

30 Young John Allen: Life of Martin Luther, Shanghai 1923, S. 1.

31 Ebd., S. 5.

32 Zhang Pinxing (Hg.): Gesamtwerk von Liang Qichao, Bd. 3: Zur Erneuerung des Volks, S. 667. 


\section{Kang Youwei, der Martin Luther des Konfuzianismus? Ein transkulturelles Beispiel}

Der Konfuzianismus war die Hauptideologie Chinas von der Han-Dynastie bis zur Qing-Dynastie und war ursprünglich eine Moralphilosophie. Es ist umstritten, ob der Konfuzianismus als Religion bezeichnet werden kann. Kang Youwei vertrat diese Ansicht und wollte den Konfuzianismus wie Martin Luther reformieren. 1891 veröffentlichte er Studien über die Fälschung der Klassiker durch die Gelebrten der Wang Mang-Periode (Xinxue Weijing Kao) und 1897 Studien über Konfuzius als Reformer (Kongzi Gaizhi Kao). Darin kritisierte Kang Youwei konfuzianische Klassiker wie etwa Liu Xin und vertrat die Auffassung, dass diese die ursprüngliche Bedeutung von Konfuzius' Lehren verzerrten. 1898 gründete Kang Youwei die konfuzianische Kirche (Kongjiao Hui) und schlug vor, den Konfuzianismus zur Staatsreligion zu machen. Sein Vorschlag wurde von der Qing-Regierung abgelehnt. Er gab nicht auf und verglich weiterhin konfuzianische Persönlichkeiten mit denen des Christentums: So nannte er 1901 Menzius den „Paulus des Konfuzianismus“.33

Feinde, Studenten und Kollegen Kang Youweis gleichermaßen sahen in ihm ein Pendant zu Martin Luther und verwendeten das Lutherbild zur Bewertung des Chinesen. Dies ist ein typisches interkulturelles Phänomen.

Ye Dehui etwa, einer seiner Gegner, verglich als erster Kang Youwei mit Martin Luther, den er dafür kritisierte, die Schriften konvertiert und Spaltungen und Streitigkeiten zwischen den Sekten geschaffen zu haben.

Kang Youwei glaubte, dass er wie Martin Luther ist, der die Kirche reformierte und die Kirche in ihren ursprünglichen Zustand zurückversetzte. Um die Sechs Klassischen Werke (Liu Jing) zu verfälschen, schrieb er zunächst Studien über die Fälschung der Klassiker durch die Gelehrten der Wang Mang-Periode (Xinxue Weijing Kao); um das politische System des Landes zu stören, schrieb er Studien über Konfuzius als Reformer (Kongzi Gaizhi Kao). Sein Gesicht ist wie ein Konfuzianer, aber sein Herz ist fremd. ${ }^{34}$

Kang Youwei war nämlich der Meinung, dass die konfuzianischen Alttexte (Gu Wen Jing) durch den Gelehrten Liu Xin (ca. 46 v. Chr. - 23 n. Chr.) gefälscht worden seien, um die Neutexte zu verdrängen, welche, Kang Youwei zufolge, die wahre Lehre des Konfuzius wiedergeben würden. ${ }^{35}$ Sowohl Luther als auch Kang Youwei suchten nach der ursprünglichen Bedeutung ihres jeweiligen Glaubens. Ye Dehui, so zeigt sich, hatte sich mit Martin Luther und seiner Wirkung intensiv auseinandergesetzt, so dass er Kang Youweis Verhalten dazu in Beziehung setzen und kritisieren konnte.

33 Kang Youwei: Gesamtwerke von Kang Youwei, Bd. 5, hg. von Jiang Yihua / Zhang Ronghua, Peking 2007, S. 411.

34 Ye Dehui: Serie der Verteidigung des Konfuzianismus (Yi Jiao Cong Bian), hg. von Shen Yunlong, Taipei 1967, S. 409-410.

35 Vgl. Wolfgang Ommerborn u. a.: Das Buch Mengzi im Kontext der Menschenrechtsfrage, Berlin 2011, S. 590. 
1901 veröffentlichte Liang Qichao eine Biografie Kang Youweis und bezeichnete ihn als „Martin Luther des Konfuzianismus“. Tan Sitong verglich 1897 in Lehre von der Menschlichkeit (Ren Xue) das Schicksal des Konfuzianismus und des Christentums: „Nach dem Aufstieg der lutherischen Religionsreform begann die Macht des Papstes nachzulassen. Die Menschen verstehen daher die wahren Lehren des Christentums. Der Niedergang des Christentums wurde vom Papst verursacht. Die Wiederbelebung des Christentums ist das Verdienst Luthers. "36

Aus der transkulturellen Perspektive können wir diese Erscheinung erläutern: Luther selbst war ein vielschichtiges Individuum und es ist schwierig, ihn vollständig zu verstehen oder zu erfassen. Jedes Bild von ihm hat eine Text- oder Tatsachengrundlage, zeigt aber meist nur einen Ausschnitt. Menschen unterschiedlichen Glaubens, unterschiedlicher Kulturen und in unterschiedlichen Positionen werden diejenige Perspektive wählen, die es ihnen erlaubt, das für sie jeweils opportune Lutherbild zu zeichnen.

\section{Fazit}

Der konfessionelle Gegensatz zwischen den christlichen Kirchen schwächte sich in der Aufklärung zunehmend ab, als Luther zum Vorkämpfer für das Recht der Vernunft und für die Gewissensfreiheit aufgewertet wurde; der deutschen Klassik und dem deutschen Idealismus erschien Luther gar als Apostel der Freiheit. In China wandelte sich das Lutherbild im ausgehenden 19. Jahrhundert: Um das absolutistische China zu reformieren und es in eine konstitutionelle Monarchie zu überführen, suchten Kang Youwei und Liang Qichao nach Vorbildern. Luthers Mut im Kampf mit dem Papst wurde ebenso betont wie seine Bedeutung als Nationalheld.

Grundsätzlich sind die Unterschiede zwischen der Reformation und der WuxuReform erheblich. Die historische und kulturelle Ausgangslage war in Deutschland und China jeweils eine völlig andere. Die Reformation in Deutschland war ein geistlicher und gesellschaftlicher Prozess. Luthers Reformation wurde vom Kaiser abgelehnt und trug zur Schwächung der kaiserlichen Autorität bei. Die WuxuReform in China war hingegen politisch initiiert und bedurfte der Hilfe des Kaisers.

Aber es gab auch einige Ähnlichkeiten: Deutschland war zu Luthers Zeit gespalten und vom Papst unterdrückt. Zu der Zeit, als Luthers Verehrung als Nationalheld begann, war Deutschland ebenfalls gespalten und von Frankreich unterdrückt. Ganz ähnlich war China von Japan besiegt worden. Beide Nationen brauchten eine Reform und eine Leitfigur. Indem das Lutherbild den jeweiligen

36 Tan Sitong: Lehre von der Menschlichkeit, in: Gesammelte Werke von Tan Sitong, hg. von Li Ao, Tianjing 2016, S. 48. 
Umständen angepasst wurde, konnte die Heldenfigur für verschiedene Ziele instrumentalisiert werden. So bietet Kang Youwei als ,Martin Luther des Konfuzianismus' ein prägnantes Beispiel für die kulturelle Interaktion zwischen China und Deutschland. 